\title{
Substrate Integrated Components for Passive Millimeterwave-Frequency Beamforming Networks
}

\author{
Laura Van Messem, Arno Moerman, Olivier Caytan, Igor Lima de Paula, Bram Hoflack, Bram Stroobandt, \\ Sam Lemey and Hendrik Rogier \\ IDLab-Electromagnetics group, imec-Ghent University (Laura.VanMessem@UGent.be)
}

\begin{abstract}
In this paper, we propose passive millimeterwave (mmWave) components for Butler matrix beamforming networks implemented in grounded-coplanar waveguide (GCPW) technology designed for integration and co-optimization with an antenna array operating in the $28 \mathrm{GHz} \mathbf{5 G}$ band. Within the frequency band of interest, covering the $[26.5 \mathrm{GHz}-29.5 \mathrm{GHz}]$ spectrum, the insertion loss of the designed hybrid coupler and crossover stays well below $0.75 \mathrm{~dB}$, while the amplitude imbalance remains below $0.5 \mathrm{~dB}$ and the phase imbalance does not exceed $5^{\circ}$.
\end{abstract}

Index Terms-Hybrid coupler, Crossover, GCPW, Multi-layer PCB, mmWave, 5G, Beamforming

\section{INTRODUCTION}

Nowadays, we see that an evolution towards the less crowded millimeterwave (mmWave) spectrum for the nextgeneration $5 \mathrm{G}$ or beyond $5 \mathrm{G}$ communication networks is inevitable to satisfy customer demands for omnipresent, data hungry and high data rate applications. At these high frequencies, antennas are typically deployed in array configurations to compensate for the increased path loss. In addition, the directive beam pattern of these antenna arrays can be electronically steered towards the users, or Multiple Input/Multiple Output (MIMO) communication schemes can be leveraged [1]. To this end, a low loss beamforming network is required that fits within the footprint of the antenna array, in which the inter antenna element spacing is typically half a wavelength for a good grating-lobe-free scan range [2].

Minimizing interconnection loss is crucial in achieving efficient mmWave systems. However, microstrip line feeding networks suffer from increased radiation loss when migrating to higher frequencies [2]-[4], while their substrateintegrated-waveguide (SIW) counterparts are generally bulky [5], [6]. Therefore, this paper describes low loss, compact components for beamforming networks based on groundedcoplanar waveguide (GCPW) technology, which is known for its compact footprint and reduced radiation characteristic, while maintaining decent power handling capability [1]. More specifically, as crucial elements for a Butler matrix beamforming network [5], an efficient GCPW-based hybrid coupler and multi-layer crossover were designed. To the best of the authors' knowledge, components for a mmWave GCPW Butler matrix implementation manufactured in standard printed circuit board (PCB) technology have not yet been pursued.

Many directional couplers in literature are developed with a designated power ratio [7]. However, for the envisaged Butler matrix beamforming network, an equal power division is necessary. The suggested hybrid coupler minimizes amplitude imbalance and adopts a bent topology, which relaxes detrimental effects caused by fabrication tolerances.

Popular planar crossover designs usually consist of concatenating two hybrid couplers, resulting in bulky structures with increased insertion losses [2], [8]. A multi-layer crossover with transmission lines on separate PCB layers provides a compact, low loss alternative next to yielding excellent crosstalk suppression.

Section II describes the design of the proposed hybrid coupler and crossover, briefly stating the operation principle, followed by the fabrication technology. The comparison between simulations and measurements is discussed in section III.

\section{Component Design}

\section{A. Design Specifications}

The passive beamforming components discussed in this contribution are tailored towards integration with the airfilled substrate integrated waveguide (AFSIW) cavity-backed patch antenna array presented in [9]. The proposed hybrid coupler and multi-layer crossover with annotated dimensions are presented in Figs. 1 and 2, respectively.

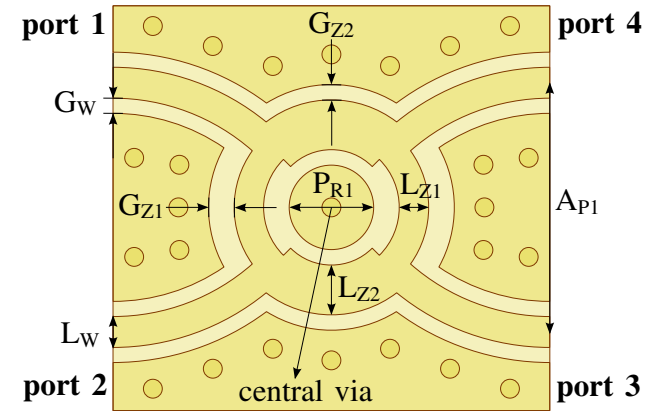

Fig. 1. Hybrid coupler design with via diameter $=0.25 \mathrm{~mm}$, via spacing $=$ $0.5 \mathrm{~mm}, \mathrm{~L}_{\mathrm{W}}=0.41 \mathrm{~mm}, \mathrm{G}_{\mathrm{W}}=0.2 \mathrm{~mm}, \mathrm{~L}_{\mathrm{Z} 1}=0.39 \mathrm{~mm}, \mathrm{G}_{\mathrm{Z} 1}=0.34 \mathrm{~mm}$, $\mathrm{L}_{\mathrm{Z} 2}=0.66 \mathrm{~mm}, \mathrm{G}_{\mathrm{Z} 2}=0.2 \mathrm{~mm}, \mathrm{P}_{\mathrm{R} 1}=1.12 \mathrm{~mm}$ and $\mathrm{A}_{\mathrm{P} 1}=3.3 \mathrm{~mm}$.

Minimizing the amplitude imbalance, while maintaining a stable $90^{\circ}$ phase difference between both output ports is the main objective when designing the hybrid coupler.

Excellent crosstalk suppression between signal paths is essential for optimal beamforming networks. Since both signal paths of the multi-layer crossover will not necessarily consist of the same electrical length, special attention is devoted to preserving an equal signal phase at the output ports. 


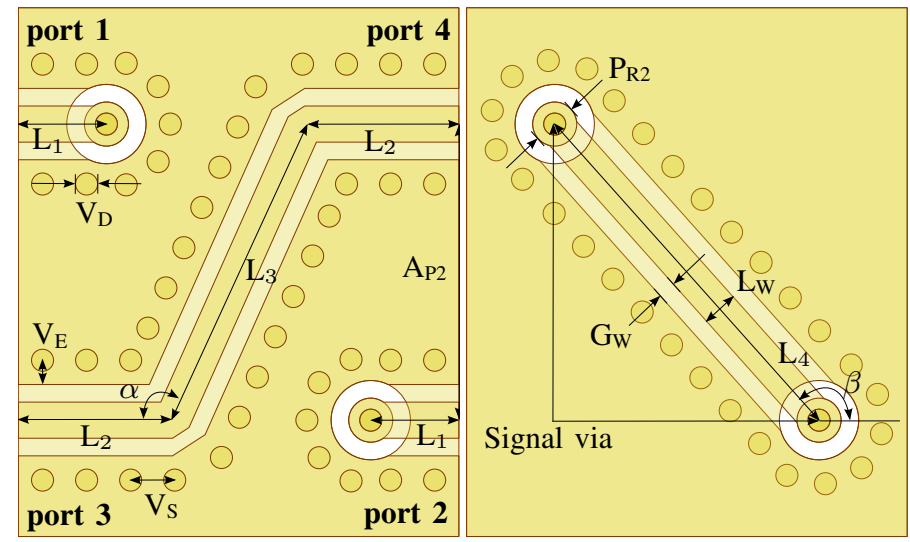

Fig. 2. Multi-layer crossover: top (left) and bottom (right) copper layer with $\mathrm{V}_{\mathrm{D}}=0.25 \mathrm{~mm}, \mathrm{~V}_{\mathrm{E}}=0.15 \mathrm{~mm}, \mathrm{~V}_{\mathrm{S}}=0.5 \mathrm{~mm}, \mathrm{~L}_{1}=1 \mathrm{~mm}, \mathrm{~L}_{2}=1.75 \mathrm{~mm}$, $\mathrm{L}_{3}=3.67 \mathrm{~mm}, \mathrm{~L}_{4}=4.5 \mathrm{~mm}, \mathrm{~L}_{\mathrm{W}}=0.41 \mathrm{~mm}, \mathrm{G}_{\mathrm{W}}=0.2 \mathrm{~mm}, \mathrm{P}_{\mathrm{R} 2}=0.5 \mathrm{~mm}$, $\mathrm{A}_{\mathrm{P} 2}=3.35 \mathrm{~mm}, \alpha=114^{\circ}$ and $\beta=132^{\circ}$. A grounding copper layer in the middle is present to minimize crosstalk.

\section{B. Operation Principle}

The components are implemented in GCPW technology, which yields minimal radiation losses and maintains decent power handling capabilities. Additional novelty of the proposed hybrid coupler lies in bending the classic square topology [7], which allows scaling to higher frequencies without introducing fabrication difficulties concerning the required copper pad around each via for full GCPW realization. Additionally, signals can easily be routed to the hybrid coupler under any incoming angle, since the feed lines also exhibit bends. Conventional hybrid branch-line couplers typically make use of two sets of transmission lines (TMLs) with a characteristic impedance of $Z_{0}=50 \Omega$ and $Z_{0} / \sqrt{2}=35.36 \Omega$. Full-wave optimization using CST Microwave Studio results in characteristic impedances of $\mathrm{Z}_{0}=55 \Omega$ and $\mathrm{Z}_{0} / \sqrt{2}=38.9 \Omega$ for the TMLs. Adding the central via (Fig. 1) is essential for a full GCPW realization.

The multi-layer crossover, shown in Fig. 2, consists of two separate GCPWs, which are maximally shielded from each other by introducing dense fences of grounding vias on each side of the copper ground, located in between both PCB layers. Ports 1 and 2 (Fig. 2, left) are connected by a GCPW section located at the bottom PCB (Fig. 2, right), which crosses the GCPW connecting ports 3 and 4 . The bottom GCPW section is reached through coax-like transition structures. Since one of the lines locally crosses over to another PCB layer with a ground plane in between, the crosstalk between both lines is minimal. Moreover, the rotational symmetry of the coax-like transition structure adds an additional degree of freedom to the design, being the bending angle $\beta$ of the GCPW line from port 1 to port 2, clarified by Fig. 2 on the right. This degree of freedom is exploited to ensure that both GCPWs are optimally phase matched across the frequency band of interest.

\section{Fabrication Technology}

The designs are implemented on a $0.254 \mathrm{~mm}$-thick RO4350B substrate $\left(\varepsilon_{r}=3.66\right.$ and $\left.\tan \delta=0.0037\right)$ with $18 \mu \mathrm{m}$-thick conductor layers. Standard PCB manufacturing is used for the fabrication of the prototypes. The two PCB layers for the crossover are assembled by adding an appropriate amount of solder paste on the via pads of the signal vias in the middle of the coax-like structure, indicated on the right of Fig. 2. Both layers are then temporarily fastened with brass screws of $1 \mathrm{~mm}$ diameter when using the heatgun to reflow the solder paste locally. Finally, the screws are removed and the galvanic contact between both PCB layers is verified.

\section{Simulation And Measurement Results}

An extended feed line is added for measurement purposes to let transition phenomena caused by the capacitive press-fit connector die out. Moreover, bends are introduced to accommodate for the minimal inter-connector distance of $1.5 \mathrm{~cm}$. A custom TRL calibration kit is fabricated to de-embed the prototypes to fix the final port planes as shown in Figs. 1 and 2.

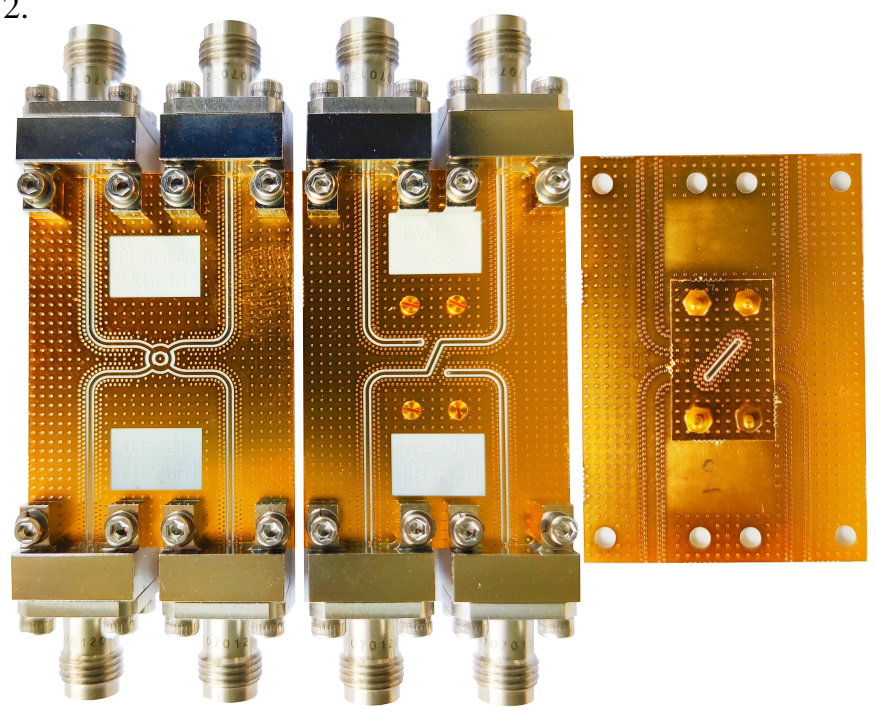

Fig. 3. Prototypes of the (left) hybrid coupler, (center) top view of the multilayer crossover prototype and (right) bottom view of the multi-layer crossover.

The simulation and measurement of the hybrid coupler are compared in Figs. 4 and 6. The simulation model predicts an insertion loss lower than $0.45 \mathrm{~dB}$ (on top of the $3 \mathrm{~dB}$ power split), whereas the measured excess insertion loss remains below $0.75 \mathrm{~dB}$. The measured maximal amplitude imbalance amounts to $0.5 \mathrm{~dB}$, which is slightly worse than the simulated maximal value of $0.25 \mathrm{~dB}$. All reflections and coupling towards the other input port remain below $-20 \mathrm{~dB}$ in simulation and measurement.

The phase difference between the output ports of the hybrid coupler is designed to be around $90^{\circ}$, which agrees well with the measured phase difference, as shown in Fig. 6. The phase imbalance remains below $1^{\circ}$ within the frequency band of interest [26.5 GHz-29.5 GHz], for both simulation and measurement.

The comparison for simulation and measurement of the multi-layer crossover is presented in Figs. 5 and 6. The design is well matched, with reflection coefficients below $-15 \mathrm{~dB}$ in simulation and measurement. In simulation, the amplitude imbalance remains within $0.15 \mathrm{~dB}$, whereas the 


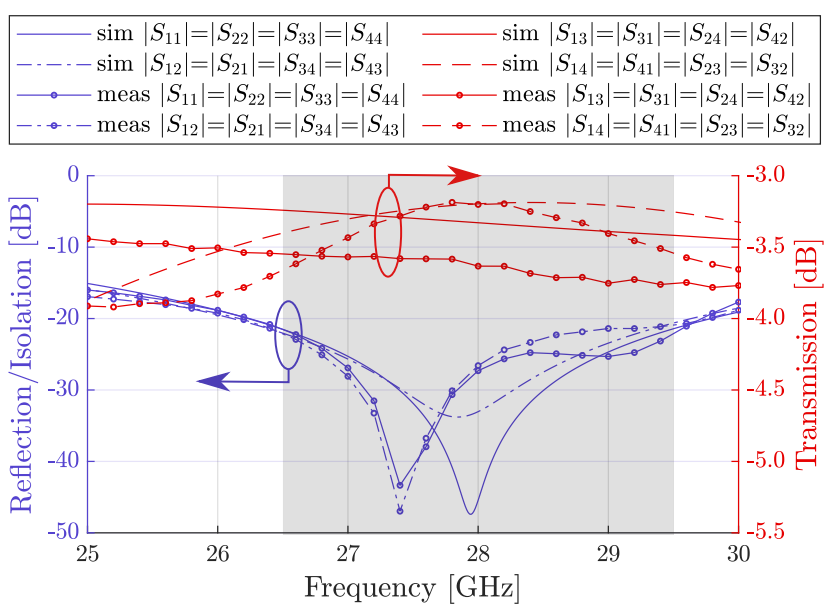

Fig. 4. Scattering parameters for the hybrid coupler: simulation (no markers) and measurement (markers).

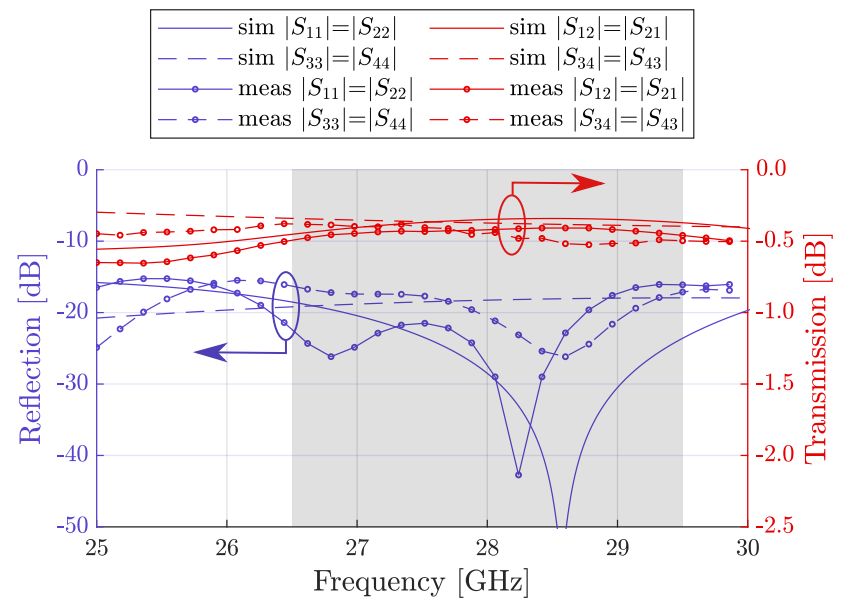

Fig. 5. Scattering parameters for the multi-layer crossover: simulation (no markers) and measurement (markers). The simulated and measured crosstalk levels remain well below $-35 \mathrm{~dB}$.

insertion losses are smaller than $0.45 \mathrm{~dB}$ within the frequency band of interest [26.5 GHz-29.5 GHz]. In measurement, a slightly worse amplitude imbalance of $0.2 \mathrm{~dB}$ and an insertion loss of $0.6 \mathrm{~dB}$ are observed. The simulated and measured crosstalk between both signal paths remains below $-35 \mathrm{~dB}$ and $-40 \mathrm{~dB}$, respectively.

The phase difference between the output ports is validated in Fig. 6. The fabricated multi-layer crossover prototype has a small airgap between both PCBs due to the soldering. Simulations show that a larger airgap increases the phase from the multi-layer path from port 1 to port 2 . To accommodate for this airgap, the initially designed phase of the indirect path (port 1 to 2 ) is $25^{\circ}$ smaller than for the direct path (port 3 to 4 ), giving some leeway to the fabricated prototypes' output port phase difference. A consistent fabrication technique resulted in an airgap around $2 \mu \mathrm{m}$ between both PCBs. Measurements with an airgap of $2 \mu \mathrm{m}$ correspond to a phase increase of $4^{\circ}$ for the multi-layer path, which agrees with the simulation for the same airgap, as can be verified by comparing the top two curves in Fig. 6 . The phase imbalance remains within $5^{\circ}$ within the $28 \mathrm{GHz} 5 \mathrm{G}$ frequency band for both simulation and measurement.

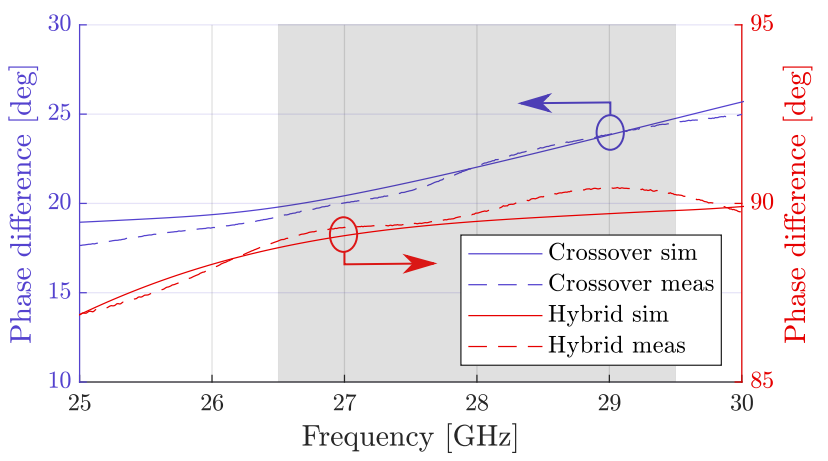

Fig. 6. Phase difference between two output ports for the multi-layer crossover with an airgap of $2 \mu \mathrm{m}$ between both PCBs (port 2 and 4 in Fig. 2, left y-axis) and the hybrid coupler (port 3 and 4 in Fig. 1, right y-axis).

\section{CONCLUSION}

A planar hybrid coupler and multi-layer crossover were designed and fabricated in grounded-coplanar waveguide (GCPW) technology. The measured insertion losses remain smaller than $0.75 \mathrm{~dB}$ within the frequency band of interest [26.5 GHz-29.5 GHz], while isolation and reflections are below $-15 \mathrm{~dB}$. The measured amplitude imbalance is maximally $0.5 \mathrm{~dB}$, while the phase imbalance does not exceed $5^{\circ}$. The design of a full Butler matrix for beamsteering applications will greatly benefit from these high performance components.

During the conference, the realization of the full Butler matrix beamforming network will be presented, integrated and co-optimized with a highly efficient, AFSIW cavity-backed patch antenna array.

\section{REFERENCES}

[1] K. Wu, M. Bozzi and N. J. G. Fonseca, "Substrate integrated transmission lines: review and applications," IEEE Journal of Microwaves, vol. 1 , no. 1, pp. 345-363, winter 2021.

[2] K. Y. Kapusuz and U. Oguz, "Millimeter wave phased array antenna for modern wireless communication systems," 2016 10th European Conference on Antennas and Propagation (EuCAP), 2016, pp. 1-4.

[3] C. Chang, R. Lee and T. Shih, "Design of a beam switching/steering Butler matrix for phased array system," IEEE Transactions on Antennas and Propagation, vol. 58, no. 2, pp. 367-374, Feb. 2010.

[4] W. F. Moulder, W. Khalil and J. L. Volakis, " $60-\mathrm{GHz}$ two-dimensionally scanning array employing wideband planar switched beam network," IEEE Antennas and Wireless Propagation Letters, vol. 9, pp. 818-821, Aug. 2010.

[5] J. Lian, Y. Ban, C. Xiao and Z. Yu, "Compact substrate-integrated 4x8 Butler matrix with sidelobe suppression for millimeter-wave multibeam application," IEEE Antennas and Wireless Propagation Letters 17, no. 5, pp. 928-932, 2018.

[6] W. M. Dyab, A. A. Sakr and K. Wu, "Dually-polarized Butler matrix for base stations with polarization diversity," IEEE Transactions on Microwave Theory and Techniques, vol. 66, no. 12, pp. 5543-5553, Dec. 2018.

[7] A. A. Abdulbari et al., "A review of hybrid couplers: state-of-theart, applications, design issues and challenges," International Journal of Numerical Modelling: Electronic Networks, Devices and Fields, e2919, early access, doi: 10.1002/jnm.2919.

[8] T. A. Denidni and M. Nedil, "Experimental investigation of a new Butler matrix using slotline technology for beamforming antenna arrays," IET Microwaves, Antennas \& Propagation, vol. 2, no. 7, pp. 641-649, Oct. 2008.

[9] I. Lima de Paula et al., "Cost-effective high-performance air-filled SIW antenna array for the global 5G $26 \mathrm{GHz}$ and $28 \mathrm{GHz}$ Bands," IEEE Antennas and Wireless Propagation Letters, vol. 20, no. 2, pp. 194-198, Feb. 2021. 